PROCEEDINGS OF THE

AMERICAN MATHEMATICAL SOCIETY

Volume 132, Number 12, Pages 3463-3466

S 0002-9939(04)07339-3

Article electronically published on July 22, 2004

\title{
A CANCELLATION LAW FOR PARTIALLY ORDERED SETS AND $T_{0}$ SPACES
}

\author{
B. BANASCHEWSKI AND R. LOWEN
}

(Communicated by Alan Dow)

\begin{abstract}
In this paper we prove a cancellation law in the category (1) of partially ordered sets and (2) of $T_{0}$ spaces, the respective conditions on the common factor being that it have (1) an irreducible bottom element and (2) an irreducible bottom element in its specialization order.
\end{abstract}

\section{INTRODUCTION}

In any category with products one has the naturally arising:

Cancellation Problem: When does $X \times Z \cong X \times Y$ imply $Z \cong Y$.

Such a property is not evident as a few examples easily show. The following products are all homeomorphic but the non-common factors are not:

$$
[0,1) \times[0,1] \approx[0,1) \times[0,1) \approx[0,1) \times(0,1) .
$$

Similarly, for any $n, m \in \mathbb{N}$ we have that $\mathbb{R}^{n} \times \mathbb{R}^{\mathbb{N}} \approx \mathbb{R}^{m} \times \mathbb{R}^{\mathbb{N}}$ but of course, if $m \neq n$, then $\mathbb{R}^{n} \not \approx \mathbb{R}^{m}$. It is well known that at present the cancellation problem, in most categories, does not have a complete answer. We refer to [1], 2], 3], [4] for several partial answers for topological spaces.

Here we are considering the above problem in the categories of partially ordered sets and finite meet and join preserving maps and of $T_{0}$ spaces and continuous maps. In the following, an element $\gamma$ in a partially ordered set will be called irreducible if, for any $\alpha$ and $\beta$, we have $\gamma=\alpha \wedge \beta$ implies $\gamma=\alpha$ or $\gamma=\beta$. Furthermore, recall that, in any topological space, the specialization order $x \leq y$ is the relation "for all open $U: x \in U$ implies $y \in U$ " or, alternatively, $x \in \bar{y}$. Note that this is a preorder which is a partial order if and only if the space is $T_{0}$. For sets $A$ and $B$ we denote by $\operatorname{pr}_{A}$ the projection $A \times B \longrightarrow A$.

\section{Cancellation Results}

We have the following cancellation result in the category of partially ordered sets and finite meet and join preserving functions.

Received by the editors September 26, 2002 and, in revised form, May 7, 2003.

2000 Mathematics Subject Classification. Primary 06A06, 54B10.

Key words and phrases. Cancellation, product, partially ordered set, $T_{0}$ space, specialization order.

(C)2004 American Mathematical Society 
2.1. Theorem. Let $X, Y$ and $L$ be partially ordered sets. Assume that $L$ has an irreducible bottom element 0 . Then any isomorphism

$$
\varphi: X \times L \longrightarrow Y \times L
$$

determines an isomorphism $\psi: X \longrightarrow Y$ where for all $x \in X$ :

$$
\psi(x)=\operatorname{pr}_{Y} \circ \varphi\left(x, \operatorname{pr}_{L} \circ \varphi(x, 0)\right) .
$$

Proof. For simplicity in notation we denote by $\varphi_{Y}$ and $\varphi_{L}$ the component maps of $\varphi$, i.e. $\varphi_{Y}:=\operatorname{pr}_{Y} \circ \varphi$ and $\varphi_{L}:=\operatorname{pr}_{L} \circ \varphi$ and analogously for $\varphi^{-1}$, i.e. $\varphi_{X}^{-1}:=\operatorname{pr}_{X} \circ \varphi^{-1}$ and $\varphi^{-1}{ }_{L}:=\operatorname{pr}_{\mathrm{L}} \circ \varphi^{-1}$.

By definition it is clear that $\psi$ is finite meet and join preserving, so we only need to prove that it has a finite meet and join preserving inverse. Hereto we also consider the map $\xi: Y \longrightarrow X$ where for all $y \in Y$,

$$
\xi(y)=\varphi_{X}^{-1}\left(y, \varphi^{-1}(y, 0)\right) \text {. }
$$

We will now prove that $\psi$ and $\xi$ are mutually inverse.

Let $(y, \alpha):=\varphi(x, 0)$, and then let $\left(p_{x}, \beta\right):=\varphi^{-1}(y, 0)$. It follows that

$$
\left(p_{x}, \beta\right)=\varphi^{-1}(y, 0) \leq \varphi^{-1}(y, \alpha)=(x, 0),
$$

which implies that $p_{x} \leq x$ and $\beta=0$. Consequently $\varphi\left(p_{x}, 0\right)=(y, 0)$.

Now set $(q, \gamma):=\varphi\left(p_{x}, \alpha\right)$. Since $\left(p_{x}, \alpha\right) \wedge(x, 0)=\left(p_{x}, 0\right)$, we have

$$
(q, \gamma) \wedge(y, \alpha)=\varphi\left(p_{x}, \alpha\right) \wedge \varphi(x, 0)=\varphi\left(p_{x}, 0\right)=(y, 0),
$$

and it follows from this that also $\gamma \wedge \alpha=0$. Now, if $\alpha \neq 0$, then it follows from the irreducibility of 0 that $\gamma=0$, and if $\alpha=0$, likewise, it follows from

$$
(q, \gamma)=\varphi\left(p_{x}, \alpha\right)=\varphi\left(p_{x}, 0\right)=(y, 0)
$$

that $\gamma=0$. Hence it follows that $\varphi\left(p_{x}, \alpha\right)=(q, 0)$. Moreover, from

$$
(y, 0)=\varphi\left(p_{x}, 0\right) \leq \varphi\left(p_{x}, \alpha\right)=(q, 0)
$$

it then follows that $y \leq q$.

Since $\left(p_{x}, \alpha\right) \vee(x, 0)=(x, \alpha)$, we have

$$
\begin{aligned}
(q, \alpha) & =(q, 0) \vee(y, \alpha) \\
& =\varphi\left(p_{x}, \alpha\right) \vee \varphi(x, 0) \\
& =\varphi(x, \alpha) \\
& =\left(\varphi_{Y}(x, \alpha), \varphi_{L}(x, \alpha)\right) \\
& =\left(\varphi_{Y}\left(x, \varphi_{L}(x, 0), \varphi_{L}(x, \alpha)\right)\right. \\
& =\left(\psi(x), \varphi_{L}(x, \alpha)\right) .
\end{aligned}
$$

Hence $q=\psi(x)$ and $\alpha=\varphi_{L}(x, \alpha)$. We deduce that

$$
\varphi\left(p_{x}, \alpha\right)=(q, 0)=(\psi(x), 0) \quad \text { and } \quad \varphi(x, \alpha)=(q, \alpha)=(\psi(x), \alpha) .
$$

We deduce that

$$
\begin{aligned}
\xi(\psi(x)) & =\varphi^{-1}{ }_{X}\left(\psi(x), \varphi^{-1}{ }_{L}(\psi(x), 0)\right) \\
& =\varphi^{-1}{ }_{X}(\psi(x), \alpha) \\
& =\operatorname{pr}_{X} \circ \varphi^{-1}(\varphi(x, \alpha)) \\
& =\operatorname{pr}_{X}(x, \alpha)=x .
\end{aligned}
$$


Hence, $\xi \circ \psi=1_{X}$. Interchanging the roles of $\varphi$ and $\varphi^{-1}$ and of $X$ and $Y$, in a symmetric fashion we conclude that $\psi \circ \xi=1_{Y}$. Consequently $\psi$ and $\xi$ are mutually inverse.

The following result is an immediate consequence.

2.2. Corollary. Let $X, Y$ and $L_{1}, \ldots, L_{n}$ be partially ordered sets. Assume that each $L_{i}$ has an irreducible bottom element. Then if $X \times L_{1} \times \cdots \times L_{n}$ is isomorphic to $Y \times L_{1} \times \cdots \times L_{n}$, then $X$ is isomorphic to $Y$.

Note that the above space $L_{1} \times \cdots \times L_{n}$ does not have an irreducible bottom element in its specialization order whenever $n \geq 2$.

The foregoing results of course have duals, where a join-irreducible largest element is considered instead of an irreducible smallest element.

2.3. Corollary. Let $X, Y$ and $L$ be partially ordered sets. Assume that $L$ has a join-irreducible largest element 1 . Then any isomorphism

$$
\varphi: X \times L \longrightarrow Y \times L
$$

determines an isomorphism $\psi: X \longrightarrow Y$ where for all $x \in X$ :

$$
\psi(x)=\operatorname{pr}_{Y} \circ \varphi\left(x, \operatorname{pr}_{L} \circ \varphi(x, 1)\right) .
$$

2.4. Corollary. Let $X, Y$ and $L_{1}, \ldots, L_{n}$ be partially ordered sets. Assume that each $L_{i}$ has a join-irreducible largest element. Then if $X \times L_{1} \times \cdots \times L_{n}$ is isomorphic to $Y \times L_{1} \times \cdots \times L_{n}$, then $X$ is isomorphic to $Y$.

We now consider the topological version of the above theorem by associating with any $T_{0}$ space its specialization order.

2.5. Theorem. Let $X, Y$ and $L$ be $T_{0}$ spaces. Assume that $L$ has an irreducible bottom element 0 in its specialization order. Then any homeomorphism

$$
\varphi: X \times L \longrightarrow Y \times L
$$

determines a homeomorphism $\psi: X \longrightarrow Y$ where for all $x \in X$ :

$$
\psi(x)=\operatorname{pr}_{Y} \circ \varphi\left(x, \operatorname{pr}_{L} \circ \varphi(x, 0)\right) .
$$

Proof. First, we have to verify that $\varphi$ determines an order isomorphism when $X \times L$ and $Y \times L$ are equipped with their specialization orders. Since $\varphi$ is a bijection, it suffices to verify that both $\varphi$ and its inverse are finite meet and join preserving. This however follows immediately from the fact that the specialization order on the product $X \times L$ (resp. $Y \times L)$ is the product of the specialization orders on $X$ and $L$ (resp. $Y$ and $L$ ), and from the continuity of $\varphi$ and its inverse. Second, we have to verify that $\psi$ and its inverse $\xi$ are continuous, but this follows at once from the formulas defining them.

Now we have the following corollaries.

2.6. Corollary. Let $X, Y$ and $L_{1}, \ldots, L_{n}$ be $T_{0}$ spaces. Assume that each $L_{i}$ has an irreducible bottom element in its specialization order. Then if $X \times L_{1} \times \cdots \times L_{n} \approx$ $Y \times L_{1} \times \cdots \times L_{n}$, then $X \approx Y$.

Note that the above space $L_{1} \times \cdots \times L_{n}$ does not have an irreducible bottom element in its specialization order whenever $n \geq 2$. 
In the following considerations $\$$ stands for the two-point Sierpinski space.

2.7. Corollary. For $T_{0}$ spaces $X$ and $Y$, if $X \times \$ \approx Y \times \$$, then $X \approx Y$.

This last corollary carries the heart of the proof of the main results. Consider $X=Y=\$$. Then

$$
\varphi: X \times \$ \longrightarrow Y \times \$:(x, \alpha) \mapsto(\alpha, x)
$$

obviously is a homeomorphism that does not restrict to a homeomorphism between $X$ and $Y$. What happens is what we could call a "Sierpinksi-switch", in that components switch places. The proof of 2.1 shows that actually this is all that can go wrong, and what one has to do is to find new images for those points $(x, 0)$ that are mapped to $(y, \alpha)$ where $\alpha>0$. For each point $(x, 0)$ that is not mapped to $Y \times\{0\}$ the proof isolates both in $X \times L$ and in $Y \times L$ subspaces of type $\$ \times \$$ that are "flipped over". The new image of the point $(x, 0)$ then is obtained by "undoing the flip" step by step as depicted in the following diagram (note that the complete path of the arrows from $(x, 0)$ to $(\psi(x), 0)$ merely indicates an order of constructing/considering points in the proof in 2.1).

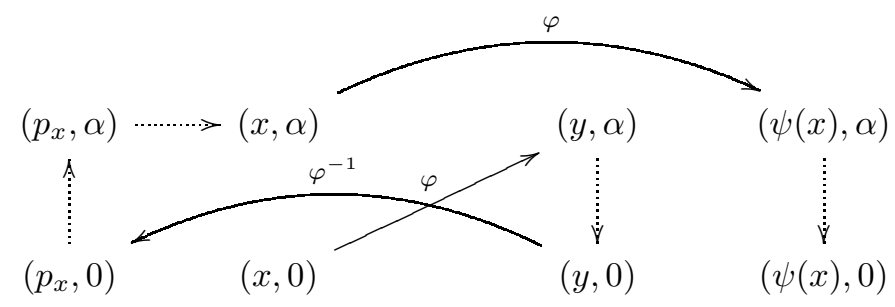

ACKNOWLEDGMENT

The authors would like to thank the anonymous referee for the suggestion to prove the result first in a purely order-theoretic context, which moreover considerably improved the presentation of the paper.

\section{REFERENCES}

[1] Behrends E. and Pelant J. The cancellation law for compact Hausdorff spaces and vectorvalued Banach-Stone theorems Arch. Math. 64 (1995) 341-343 MR 1319005 (96h:46046)

[2] Borsuk K. Sur la décomposition des polyèdres en produits cartésiens Fund. Math. 33 (1938) 137-148

[3] Fox R.H. On a problem of S. Ulam concerning cartesian products Fund. Math. 34 (1947) 278-287 MR $0027502(10: 316 a)$

[4] Zeron S.E. Cancellation laws in topological products Houston J. Math. 27 (2001) 67-74 MR 1843913 (2002d:54003)

Department of Mathematics and Statistics, McMaster University, Hamilton, Ontario, Canada L8S $4 \mathrm{~K} 1$

Department of Mathematics and Computer Science, University of Antwerp, MiddelHeimlaAn 1, 2020 ANTwerp, Belgium

E-mail address: rlow@ruca.ua.ac.be 